\title{
Remarks on bosonic super-WIMP search experiments
}

\author{
Young Ju Ko ${ }^{1, *}$ and HyangKyu Park $\oplus^{2, \dagger}$ \\ ${ }^{1}$ Center for Underground Physics, Institute for Basic Science (IBS), Daejeon 34126, Republic of Korea \\ ${ }^{2}$ Department of Accelerator Science, Korea University, Sejong 30019, Republic of Korea
}

(Received 4 June 2021; accepted 3 October 2021; published 26 October 2021)

\begin{abstract}
Bosonic super-weakly interacting massive particles (WIMPs), including pseudoscalar and vector particles, are dark-matter candidates. To date, many underground experiments searches for super-WIMPs have been performed in the mass range of a few $\mathrm{keV} / c^{2}$ to $1 \mathrm{MeV} / c^{2}$. All these searches utilize the absorption process of a super-WIMP by a target atom in the detector, which is similar to the photoelectric effect. We consider another process-namely, a Compton-like process. As an example, we compare the cross section of a germanium atom for the absorption process with that of a Compton-like process. Our findings indicate that the cross section for the Compton-like process becomes dominant relative to that for the absorption process for mass above approximately $150 \mathrm{keV} / c^{2}$ for both pseudoscalar and vector superWIMPs. In particular, the cross section for the Compton-like process for a vector super-WIMP becomes increasingly greater than that for the absorption process by 1 to 2 orders of magnitude in the $400 \mathrm{keV} / c^{2}$ to $1 \mathrm{MeV} / c^{2}$ mass range, respectively. By including the Compton-like process, which has not been used in any other super-WIMP search experiments, the experimental upper limits can be improved.
\end{abstract}

DOI: 10.1103/PhysRevD.104.083030

\section{INTRODUCTION}

One of the most fundamental questions in physics is the nature of dark matter (DM). The existence of DM, which constitutes approximately $80 \%$ of the matter in the Universe [1], has been inferred from a wide range of astrophysical and cosmological systems based solely on gravitational effects [2,3]. Although the standard model (SM) of particle physics is extraordinarily successful, it cannot explain the nature of DM.

The freeze-out mechanism is a plausible method for the generation of the DM population in our Universe. It has been assumed that DM particles, commonly called weakly interacting massive particles (WIMPs), interacted with SM particles in thermal equilibrium at very high temperatures in the early Universe, and the DM particles that were out of equilibrium remained as the Universe expanded. The currently observed dark-matter relic abundance has been generated due to the weak mass scale $\mathcal{O}(100 \mathrm{GeV})$ of DM with weak interaction strength between the WIMPs and SM particles $[4,5]$. The DM produced by this mechanism is

\footnotetext{
yjko@ibs.re.kr

Corresponding author. hyangkyu@korea.ac.kr

Published by the American Physical Society under the terms of the Creative Commons Attribution 4.0 International license. Further distribution of this work must maintain attribution to the author(s) and the published article's title, journal citation, and DOI. Funded by SCOAP .
}

commonly referred to as a WIMP. Extensive searches over the past 40 years for a WIMP with $\mathcal{O}(100 \mathrm{GeV})$ mass have not yielded any results. Hence, it is desirable to investigate various theoretical models of DM.

Based on the numerical simulation results for the smallscale structure formation of a WIMP based on gravitational interaction, the DM mass is considered to be greater than $\sim 3 \mathrm{keV}$ [6]. A mass on this scale implies weak interaction between the DM and SM particles. Several models have been proposed with $\mathrm{DM}$ mass scales ranging from $\mathrm{keV}$ to $\mathrm{MeV}$ for the so-called super-WIMP [7-12]. Fermionic super-WIMPs, sterile neutrinos, and gravitinos have been thoroughly studied and are extremely difficult to detect experimentally. On the other hand, bosonic super-WIMPs allow the decay of lighter SM particles [7], and such decays with superweak interactions can be strongly suppressed. Nonetheless, super-WIMPs can be absorbed or emitted by SM particles, which can be directly detected through terrestrial experiments. In particular, their absorption process in the target material deposits energy into the target atom, which corresponds to the rest mass of the super-WIMP.

The upper limits of the bosonic super-WIMP masses are about $300 \mathrm{keV} / \mathrm{c}^{2}$ and are estimated by comparing the gamma rays from the extragalactic halo with the expected photon flux due to the bosonic super-WIMP decays [13]. If the bosonic super-WIMPs do not constitute a significant fraction of the DM, this estimation does not give such a constraint for the upper limits of the masses. Furthermore, the masses in some extensions of the bosonic super-WIMP model can be sub-MeV/c $c^{2}[14]$ or larger than $1 \mathrm{MeV} / c^{2}$ 
[15]. If the masses of the bosonic super-WIMPs are larger than twice the electron mass, they can decay into an $e^{+} e^{-}$ pair such that their lifetime could be too short to be the DM. It would be worthwhile to search for the bosonic superWIMP mass in the range of $\mathcal{O}\left(\mathrm{MeV} / c^{2}\right)$.

Experimental explorations for pseudoscalar (vector) super-WIMPs, known as axionlike-particles (dark photons), have been performed based on their absorption in detector material. The constraints for the coupling strength of super-WIMPs with electrons for the super-WIMP mass have been determined through underground experiments [16-26], which investigate the super-WIMP mass region from $\sim \mathrm{keV} / c^{2}$ to $1 \mathrm{MeV} / c^{2}$. To detect super-WIMP events, all these studies utilize the absorption process of the super-WIMP by target atoms, which is similar to the photoelectric effect; the cross sections for this process are proportional to those of the photoelectric effect, where the photon energy is equal to the super-WIMP mass. When using the absorption process for super-WIMP detection, the upper limit becomes weak with the increase in the superWIMP mass. For example, the cross section of Ge for the photoelectric effect decreases significantly from $4306 \mathrm{~b} /$ atom at $10 \mathrm{keV}$ to $0.0086 \mathrm{~b} /$ atom at $1 \mathrm{MeV}$ for the incoming photon energy.

In this paper, we consider a Compton-like process $a+e^{-} \rightarrow e^{-}+\gamma\left(V+e^{-} \rightarrow e^{-}+\gamma\right)$ for a pseudoscalar (vector) super-WIMP, where $a$ and $V$ represent a pseudoscalar and vector super-WIMP, respectively. In general, the cross section for the Compton process is greater than that for the photoelectric effect at photon energies above $\sim 100 \mathrm{keV}$. By including the Compton-like process, we expect an improvement in the experimental sensitivity in the mass range of $\sim 100 \mathrm{keV} / c^{2}$ to a few $\mathrm{MeV} / c^{2}$. The most recent search for super-WIMPs used Ge atoms as the target material in the GERDA experiment [20] for a superWIMP mass range up to $1 \mathrm{MeV} / c^{2}$. We study the cross section for the absorption and Compton-like processes with a Ge target for a super-WIMP model in the $1 \mathrm{keV} / c^{2}$ to $1 \mathrm{MeV} / c^{2}$ mass range.

\section{THE MODEL FOR SUPER-WIMP}

In this section, we discuss the absorption process, which is similar to the photoelectric effect, and a Compton-like process for the interaction of a pseudoscalar super-WIMP and vector super-WIMP with an atom and electron, respectively. The Lagrangian for the interaction of pseudoscalar super-WIMP $a$ with an electron [7] can be expressed as follows:

$$
\mathcal{L}=2 \frac{m_{e}}{f_{a}} a \bar{\psi} i \gamma_{5} \psi
$$

where $f_{a}$ is the interaction strength, $m_{e}$ is the electron mass, and $\psi$ is the electron field. Therefore, this interaction Lagrangian gives the cross section for the absorption process $\sigma_{a, a b s}$ [7] with an atom, which is the so-called axioelectric effect, in terms of the cross section for the photoelectric effect $\sigma_{\text {pe }}$, with the photon energy $\omega$ equivalent to the mass of the pseudoscalar super-WIMP $\left(m_{a}\right)$ :

$$
\frac{\sigma_{a, a b s} v}{\sigma_{\mathrm{pe}}\left(\omega=m_{a}\right) c} \approx \frac{3 m_{a}^{2}}{4 \pi \alpha f_{a}^{2}}
$$

where $v$ is the incoming velocity of the super-WIMP, $c$ is the velocity of light, and $\alpha$ is the fine structure constant. Equation (2) can be rewritten by introducing dimensionless coupling $g_{a e e}=2 m_{e} / f_{a}$ as follows:

$$
\frac{\sigma_{a, a b s} v}{\sigma_{\mathrm{pe}}\left(\omega=m_{a}\right) c} \approx g_{a e e}^{2} \frac{3 m_{a}^{2}}{16 \pi \alpha m_{e}^{2}} .
$$

For the interaction of vector super-WIMP $V$ with an electron, the Lagrangian [7] is given by

$$
\mathcal{L}=e \kappa V_{\mu} \bar{\psi} \gamma^{\mu} \psi
$$

where $V_{\mu}$ is the field of the super-WIMP, $e$ is the electron charge, and $\kappa$ is the kinetic mixing parameter with the electromagnetic field. The absorption cross section of the vector super-WIMP $\left(\sigma_{V, a b s}\right)$ by an atom [7] is calculated as

$$
\frac{\sigma_{V, a b s} v}{\sigma_{\mathrm{pe}}\left(\omega=m_{V}\right) c} \approx \frac{\alpha^{\prime}}{\alpha}
$$

where $m_{V}$ is the mass of the vector super-WIMP and $\alpha^{\prime}$ is $\frac{(e \kappa)^{2}}{4 \pi}$.

The Compton-like process where a pseudoscalar superWIMP or vector super-WIMP interacts with an electron and produces a photon $(\gamma)$ is as follows:

$$
e+\phi \rightarrow e+\gamma,
$$

where $\phi$ can be either a pseudoscalar super-WIMP or vector super-WIMP. The cross section for the Comptonlike process of a pseudoscalar (vector) super-WIMP, $\sigma_{a, \text { cmp }}$ $\left(\sigma_{V, \mathrm{cmp}}\right)$ [27], can be determined as follows:

$$
\begin{aligned}
& \sigma_{a, \mathrm{cmp}}=g_{a e e}^{2} \frac{\alpha}{4 m_{e}|\mathbf{k}|} \int_{-1}^{1} d \cos \theta_{\gamma} \frac{|\mathbf{q}| \mathcal{A}_{P}}{E_{k}+m_{e}-|\mathbf{k}| \cos \theta_{\gamma}}, \\
& \sigma_{V, \mathrm{cmp}}=\kappa^{2} \frac{\pi \alpha^{2}}{3 m_{e}|\mathbf{k}|} \int_{-1}^{1} d \cos \theta_{\gamma} \frac{|\mathbf{q}| \mathcal{A}_{V}}{E_{k}+m_{e}-|\mathbf{k}| \cos \theta_{\gamma}},
\end{aligned}
$$

where $\mathbf{k}$ is the momentum of the incoming super-WIMP, $\mathbf{q}$ is the momentum of the outgoing photon, $\theta_{\gamma}$ is the angle between the photon and super-WIMP, and $\mathcal{A}_{P}$ and $\mathcal{A}_{V}$ are the matrix elements of the Compton-like process for the pseudoscalar super-WIMP and vector super-WIMP [27], 

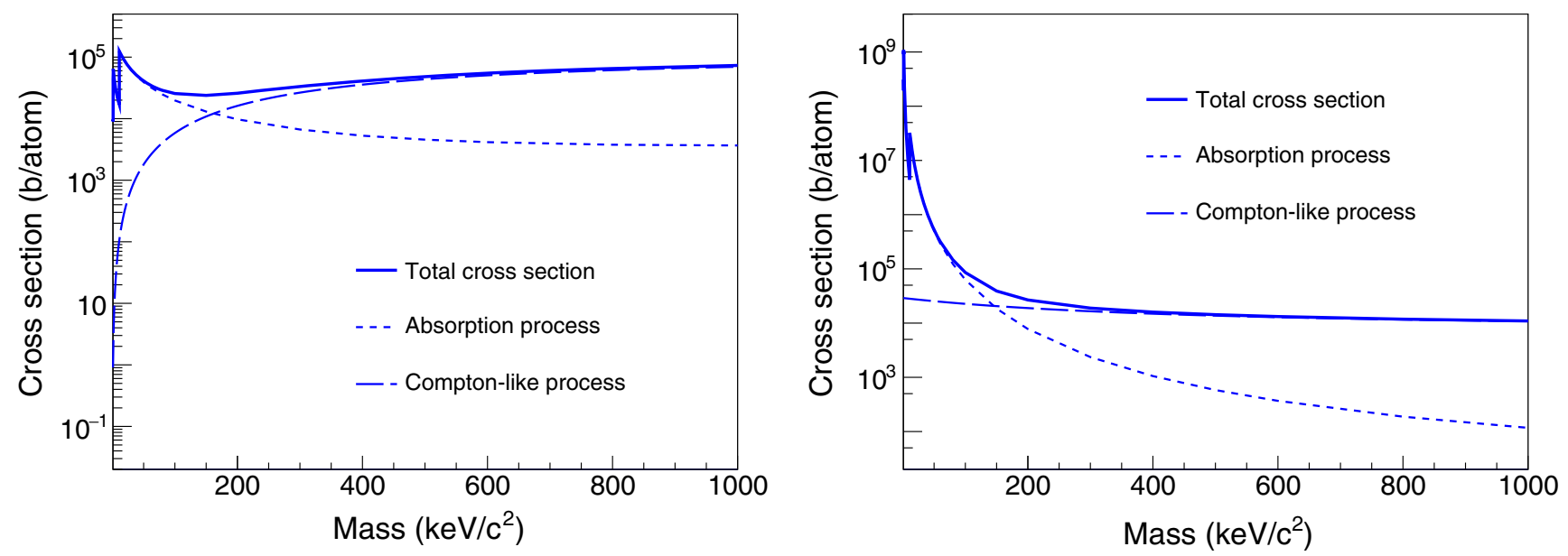

FIG. 1. Cross sections (unit, b/atom) for the absorption and Compton-like processes, and the total cross section, including both processes, for the pseudoscalar (left panel) and vector (right panel) super-WIMPs as a function of the mass. We used the dimensionless coupling constant $g_{a e e}=1$ (kinetic mixing parameter $\kappa=1$ ) for a pseudoscalar (vector) super-WIMP.

respectively. Here, $|\mathbf{q}|$ for the Compton-like process in Eqs. (7a) and (7b) is given by

$$
|\mathbf{q}|=\frac{m_{\phi}^{2}+2 E_{k} m_{e}}{2\left(m_{e}+E_{k}-|\mathbf{k}| \cos \theta_{\gamma}\right)}
$$

where $m_{\phi}$ is the mass of the super-WIMP and $E_{k}$ is the energy of the super-WIMP, which can be considered the mass of the super-WIMP.

\section{CROSS-SECTION ESTIMATIONS FOR THE ABSORPTION AND COMPTON-LIKE PROCESSES}

In this section, we discuss the cross section of the $\mathrm{Ge}$ atom for the absorption and Compton-like processes. For estimating the cross section for the absorption process, we used Eq. (3) for a pseudoscalar super-WIMP and Eq. (5) for a vector super-WIMP. For such an estimation, the cross sections for the photoelectric effect $\left(\sigma_{\mathrm{pe}}\right)$ of the Ge atoms were used in the XCOM: Photons Cross Sections Database [28].

For an atom, the cross sections of the Compton-like process for a pseudoscalar and vector super-WIMP are given by

$$
\begin{gathered}
\sigma_{a, \mathrm{cmp}}^{Z}=N_{e} \sigma_{a, \mathrm{cmp}}, \\
\sigma_{V, \mathrm{cmp}}^{Z}=N_{e} \sigma_{V, \mathrm{cmp}},
\end{gathered}
$$

where $\sigma_{a, \mathrm{cmp}}^{Z}\left(\sigma_{V, \mathrm{cmp}}^{Z}\right)$ is the cross section for the Comptonlike process for a pseudoscalar (vector super-WIMP) for an atom and $N_{e}$ is the number of electrons (atomic number) in the atom. $\sigma_{a, \mathrm{cmp}}$ and $\sigma_{V, \mathrm{cmp}}$ can be calculated numerically using Eqs. (7a) and (7b), respectively. We set $N_{e}=32$ as the atomic number of the $\mathrm{Ge}$ atom.
The total cross section $\left(\sigma_{\text {atom }}\right)$ for a super-WIMP interacting with an atom, including both the absorption and Compton-like processes, can be expressed as

$$
\sigma_{\text {atom }}=\sigma_{a(V), a b s}+\sigma_{a(V), \mathrm{cmp}}^{Z} .
$$

To compare the cross sections for the absorption and Compton-like processes for the interaction of a pseudoscalar (vector) super-WIMP with a Ge atom, we applied the dimensionless coupling constant $g_{a e e}=1$ (kinetic mixing parameter $\kappa=1$ ). The results for each process and the total cross section for the pseudoscalar and vector super-WIMP are shown in Fig. 1. As previously mentioned, the cross section for the Compton-like process is greater than that of the absorption process in the mass range above approximately $500 \mathrm{keV} / c^{2}\left(100 \mathrm{keV} / c^{2}\right)$ for a pseudoscalar (vector) super-WIMP. In particular, the cross section for the Compton-like process for a vector super-WIMP continues to increase from 1 to 2 orders of magnitude in the $400 \mathrm{keV} / c^{2}$ to $1 \mathrm{MeV} / c^{2}$ mass range, respectively.

\section{CONCLUSIONS}

In this paper, we examined the absorption and Comptonlike processes for the interaction of a super-WIMP with an atom. As an example, we compared the cross sections of both processes for a Ge atom. The obtained results demonstrated that the Compton-like process had a greater cross section than the absorption process for masses above approximately $150 \mathrm{keV} / c^{2}$ for both pseudoscalar and vector super-WIMPs. The cross section for the Compton-like process for a pseudoscalar super-WIMP is increased to 1 order of magnitude at $1 \mathrm{MeV} / c^{2}$ of mass. However, the cross section for the Compton-like process for a vector super-WIMP becomes increasingly greater than that for the absorption process by 1 to 2 orders of magnitude in the $400 \mathrm{keV} / c^{2}$ to $1 \mathrm{MeV} / c^{2}$ 
mass range, respectively. By including the Compton-like process, which has not been used in any other super-WIMP search experiments, the experimental upper limits can be improved.

\section{ACKNOWLEDGMENTS}

We acknowledge Ein Park and Euny Lee for their invaluable discussions. The work of Y. J. K. was supported by the Institute for Basic Science (IBS) in Korea under Project No. IBS-R016-A1. H. P. was supported by a Korea University grant and the National Research Foundation of Korea (Grant No. NRF-2018R1D1A1B07048941), and a Korea Basic Science Institute (KBSI) National Research Facilities and Equipment Center (NFEC) grant funded by the Korean Government (Ministry of Education) (Grant No. 2019R1A6C1010027).
[1] P. A. R. Ade et al. (Planck Collaboration), Astron. Astrophys. 594, A23 (2016).

[2] L. Bergstrom, Ann. Phys. (Berlin) 524, 479 (2012).

[3] J. L. Feng, Annu. Rev. Astron. Astrophys. 48, 495 (2010).

[4] B. W. Lee and S. Weinberg, Phys. Rev. Lett. 39, 165 (1977).

[5] M. I. Vysotsky, A. D. Dolgov, and Y. B. Zeldovich, JETP Lett. 26, 188 (1977), http://jetpletters.ru/ps/1375/article_ 20858.shtml.

[6] K. Marković and M. Viel, Pub. Astron. Soc. Aust. 31, e006 (2014).

[7] M. Pospelov, A. Ritz, and M. B. Voloshin, Phys. Rev. D 78, 115012 (2008).

[8] M. Pospelov, A. Ritz, and M. B. Voloshin, Phys. Lett. B 662, 53 (2008).

[9] S. Dodelson and L. M. Widrow, Phys. Rev. Lett. 72, 17 (1994).

[10] A. D. Dolgov and S. H. Hansen, Astropart. Phys. 16, 339 (2002).

[11] J. R. Ellis, D. V. Nanopoulos, and S. Sarkar, Nucl. Phys. B259, 175 (1985).

[12] J. L. Feng, A. Rajaraman, and F. Takayama, Phys. Rev. D 68, 063504 (2003).

[13] P. Arias, D. Cadamuro, M. Goodsell, J. Jaeckel, J. Redondo, and A. Ringwald, J. Cosmol. Astropart. Phys. 06 (2012) 013.

[14] A. Aboubrahim, W.Z. Feng, P. Nath, and Z. Y. Wang, J. High Energy Phys. 06 (2021) 086.
[15] K. Kaneta, H. S. Lee, and S. Yun, Phys. Rev. D 95, 115032 (2017).

[16] K. Abe et al. (XMASS Collaboration), Phys. Lett. B 787, 153 (2018).

[17] C. Fu et al. (PandaX Collaboration), Phys. Rev. Lett. 119, 181806 (2017).

[18] D. S. Akerib et al. (LUX Collaboration), Phys. Rev. Lett. 118, 261301 (2017).

[19] E. Aprile et al. (XENON100 Collaboration), Phys. Rev. D 96, 122002 (2017).

[20] M. Agostini et al. (GERDA Collaboration), Phys. Rev. Lett. 125, 011801 (2020).

[21] T. Aralis et al. (SuperCDMS Collaboration), Phys. Rev. D 101, 052008 (2020); 103, 039901(E) (2021).

[22] E. Armengaud et al. (EDELWEISS Collaboration), Phys. Rev. D 98, 082004 (2018).

[23] S. K. Liu et al. (CDEX Collaboration), Phys. Rev. D 95, 052006 (2017).

[24] N. Abgrall et al. (Majorana Collaboration), Phys. Rev. Lett. 118, 161801 (2017).

[25] G. Angloher et al. (CRESST Collaboration), Eur. Phys. J. C 77, 299 (2017).

[26] P. Adhikari et al. (COSINE-100 Collaboration), Astropart. Phys. 114, 101 (2020).

[27] Y. S. Liu and G. A. Miller, Phys. Rev. D 96, 016004 (2017).

[28] Database website, http://physics.nist.gov/PhysRefData/ Xcom/html/xcom1.html. 